\title{
Design and implementation of a proficiency- based, structured endoscopy course for medical students applying for a surgical specialty
}

This article was published in the following Dove Press journal:

Advances in Medical Education and Practice

8 May 2013

Number of times this article has been viewed

\author{
Gunter De Win ${ }^{1,2}$ \\ Siska Van Bruwaene \\ Christopher Allen ${ }^{3}$ \\ Dirk De Ridder ${ }^{2}$ \\ 'Centre for Surgical Technologies, \\ ${ }^{2}$ Department of Urology, University \\ Hospitals, KU Leuven, Leuven, \\ Belgium; ${ }^{3}$ School of Arts and Sciences, \\ University of Pennsylvania, PA, USA
}

Correspondence: Gunter De Win Adolescent, Paediatric, and Reconstructive Urology, University Hospital Antwerp, Wilrijkstraat 10, 2650 Edegem, Belgium

$\mathrm{Tel}+32382$ I 5586

Fax +32 382I 4479

Email gunterdewin@me.com
Background: Surgical simulation is becoming increasingly important in surgical education. Despite the important work done on simulators, simulator model development, and simulator assessment methodologies, there is a need for development of integrated simulators in the curriculum. In this paper, we describe the design of our evidence-based preclinical training program for medical students applying for a surgical career at the Centre for Surgical Technologies.

Methods: Twenty-two students participated in this training program. During their final months as medical students, they received structured, proficiency-based endoscopy training. The total amount of mentored training was 18 hours and the training was organized into three training blocks. The first block focused on psychomotor training, the second block focused on laparoscopic stitching and suturing, and the third block on laparoscopic dissection techniques and hemostasis. Deliberate practice was allowed and students had to show proficiency before proceeding to the next training block. Students' psychomotor abilities were tested before the course and after each training block. At the beginning of their careers as surgical registrars, their performance on a laparoscopic suturing task was compared with that of registrars from the previous year who did not have this training course. Student opinions about this course were evaluated using a visual analog scale.

Results: All students rated the training course as useful and their psychomotor abilities improved markedly. All students performed deliberate practice, and those who participated in this course scored significantly $(P<0.0001)$ better on the laparoscopic suturing task than first year registrars who did not participate in this course.

Conclusion: Organization of a structured preclinical training program in laparoscopy for final year medical students is feasible, attractive, and successful.

Keywords: laparoscopy training, proficiency based, surgical skill evaluation, curriculum development

\section{Introduction}

One of the most significant developments in surgery over the past 20 years has been the advent of minimally invasive laparoscopic surgery. The skills required to perform such surgery are challenging and require surgical registrars to go through an intensive learning period. While assisting their supervisor, residents are typically encouraged to observe the surgeon performing procedures on patients under general anesthesia in the operating room. This may not be the most effective teaching method, because subtle procedural knowledge about hand movements and control of instruments may not be readily noticed or understood by the student or communicated by the surgeon. To learn these skills, extensive manual training is needed. ${ }^{1,2}$ Medicolegal issues and time 
constraints now make it difficult for students to "train" on patients in theater. Owing to the limitations of this apprenticetutor model, a significant number of specialists have agreed that some form of preclinical surgical training must be done outside of the operating room.

The concept of simulation training seems to be a promising alternative to observation in the operating room. Many surgical skills centers have been created, each of which has developed its own models of instruction, from human cadavers and animal models to pulsatile organ perfusion, synthetic bench models, and drill exercises. Even virtual trainers have been developed, which incorporate direct assessment and instruction methods.

Because the ultimate goal of simulation training is to improve the surgeon's performance in theater, transferability and retention studies are extremely important. Such studies evaluate the effectiveness of simulation training over time and have shown that it is not solely the simulator, but also the manner of training on the simulator, that determines the degree of transference of skill to the operative setting. Training until proficient and deliberate practice have been shown to be important factors. ${ }^{3-6}$ However, the manner of instruction that most effectively integrates simulation into the surgical curriculum is not yet clear. ${ }^{4,7,8}$

Despite the important work done on simulators, development of simulator models, and assessment methods, there remains a need for integration of simulators into the surgical curriculum. ${ }^{9}$ Schout et al recommended designing and evaluating a comprehensive training program rather than validating only one aspect of the curriculum that can be performed on a simulator. ${ }^{10}$ However, this requires an understanding of educational theories and a multidisciplinary approach involving collaboration between educational theorists, residents, trainers, and industrial designers. Schout et al suggest that training needs should be addressed before designing a training program, after which the requirements of simulators can be investigated, with selection of appropriate models to cover the needs of training programs.

In spite of the significant amount of data available on the use and efficacy of simulation models and training parameters, a structured and validated training curriculum that incorporates different models and scientific evidence on training structure in order to prepare the student to acquire endoscopic skills before starting traditional training in the operating room has not been implemented in most training centers. Only the American Fundamentals of Laparoscopic Surgery program, which focuses predominantly on manual psychomotor skills, has been validated. ${ }^{11,12}$
The purpose of this study was to design a proficiencybased training program based on recently acquired evidence in simulation training, using different simulator models depending on training needs, and to evaluate its feasibility and efficacy. In this paper, we describe the design of our evidencebased preclinical training program at the Centre for Surgical Technologies (CST PTP), which uses inexpensive bench models, box trainers, and animal tissue. We predicted that medical students pre-trained using this program would retain some of the surgical skills they had acquired, which would be advantageous when entering training as surgical registrars.

\section{Materials and methods Training needs}

The aim of our course was to provide graduating medical students entering a surgical specialty with the basic skills necessary to learn laparoscopy as a surgical trainee in theater. It was not our intention to teach them specific surgical procedures. Before designing the training program, we identified the skill set that needed to be acquired by observing different types of laparoscopic surgery and identifying the skills common to all these procedures. An expert in motor learning from the sports department of the University of Leuven was consulted, who suggested that we begin our research by focusing on laparoscopic psychomotor skills. His ideas were combined with the skills felt to be most important in the opinion of surgical trainers. Student expectations were also incorporated.

Specific psychomotor laparoscopic skills identified were laparoscopic camera navigation with a $0^{\circ}$ and $30^{\circ}$ scope, depth perception from a two-dimensional screen using subtle visual clues, hand-eye coordination, remote handling of instruments using the dominant and non-dominant hand, operating without tactile feedback, and the fine motor skills needed to deal with the fulcrum effect and the lever forces generated by long instruments.

Our surgical trainers believed that it was also necessary to learn intracorporeal suturing at this early stage of the curriculum, not only because it increases the dexterity of the trainee, but also because one might unexpectedly need the skill of intracorporeal suturing and knot tying, even during basic procedures. Basic tissue handling, dissection, and coagulation were also identified as fundamental skills that should be acquired during an ideal preclinical course.

\section{Training program design}

The training program requirements were outlined based on existing practical and theoretical knowledge about the use of 
simulators in general. The design of the program is shown in Figure 1. The CST PTP was built up around three separate training blocks. During each block, students learned one of the identified training needs. Block 1 focused on laparoscopic psychomotor training, block 2 on intracorporeal suturing and knot tying, and block 3 on tissue handling, dissection, and hemostasis. Each training block consisted of four daily supervised and proctored lessons of 1.5 hours each (ie, distributed training). In all, each block included six contact hours, and the CST PTP lasted a total of 18 hours.

With the help of a web-based (Toledo) platform, the students needed to prepare cognitively for each lesson. ${ }^{13}$ Each exercise was explained step-by-step in a video. The scoring system of every exercise was explained in detail. The students were also made aware of the performance target. The scoring system was designed to be easy to use and allowed the students to perform self-assessment so that they could follow up their own progress (ie, structured and goal-directed training to proficiency level).
Each exercise in each lesson started with the video demonstration followed by a step-by-step skill demonstration by the teacher and an explanation of the scoring system. Afterwards, the students practiced the given exercise. During this practice session, one instructor proctored a maximum of six students, giving the students constructive feedback and correcting any wrong movements. Peers could also give feedback.

After the fourth lesson of each training block, students were expected to spend additional time practicing and refining the skills they had developed. To facilitate this, each student was given full access to the training room, laparoscopy material, and training models. An online booking system was installed to allow students to coordinate their use of the surgical training facilities and ensure that each student had the opportunity to practice the skills acquired in the previous training block. The web-based platform with video instructions was also available in the deliberate practice room to give the students the chance to imitate the video instructions.

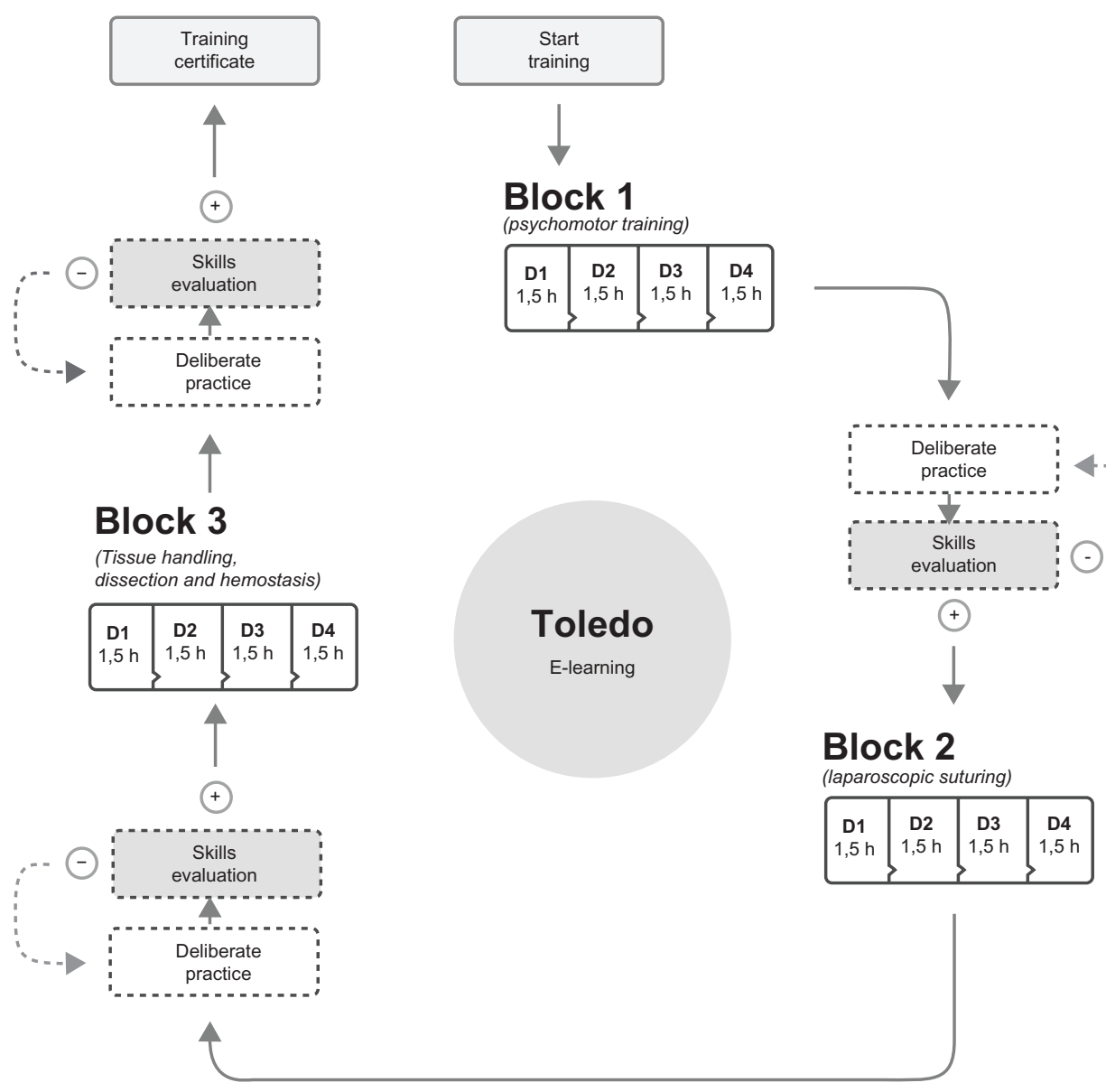

Figure I Design of the preclinical training program at the Centre for Surgical Technologies.

Notes: Every training block starts with online instructions which the student can prepare at home. Every training block consists of four daily training sessions each of 1.5 hours. At the end of each training block, the students have the opportunity to enhance their skills with performing deliberate practice. Two weeks later, the skills acquired are tested. If proficiency is reached, the trainee can continue with the next training block. 
Students could also record and review their own performance (ie, deliberate practice).

Two weeks after the final lesson of each training block, each student had to pass a skills evaluation test before starting the next teaching block. The proficiency level for each exercise was clearly communicated on the web-based platform, but the students did not know in advance which exercise would be tested. If the student did not succeed in the assigned exercise, extra feedback from the trainer and evaluator was given, and the student was asked to intensify his or her deliberate practice and to succeed in a repeat test one week later. After passing the skill test, a training certificate for the specific training block was issued and the next teaching block started (ie, proficiency reached).

\section{Training media}

\section{Block I: laparoscopic psychomotor skills}

The laparoscopic psychomotor skills training and testing (LASTT) model, suitable for performing standardized exercises in laparoscopic psychomotor training, was used ${ }^{14}$ (Figure 2). The model was inserted into the Szabo trainer box (Karl Storz, Tuttlingen, Germany). All exercises were developed and validated at our training center. ${ }^{14,15}$
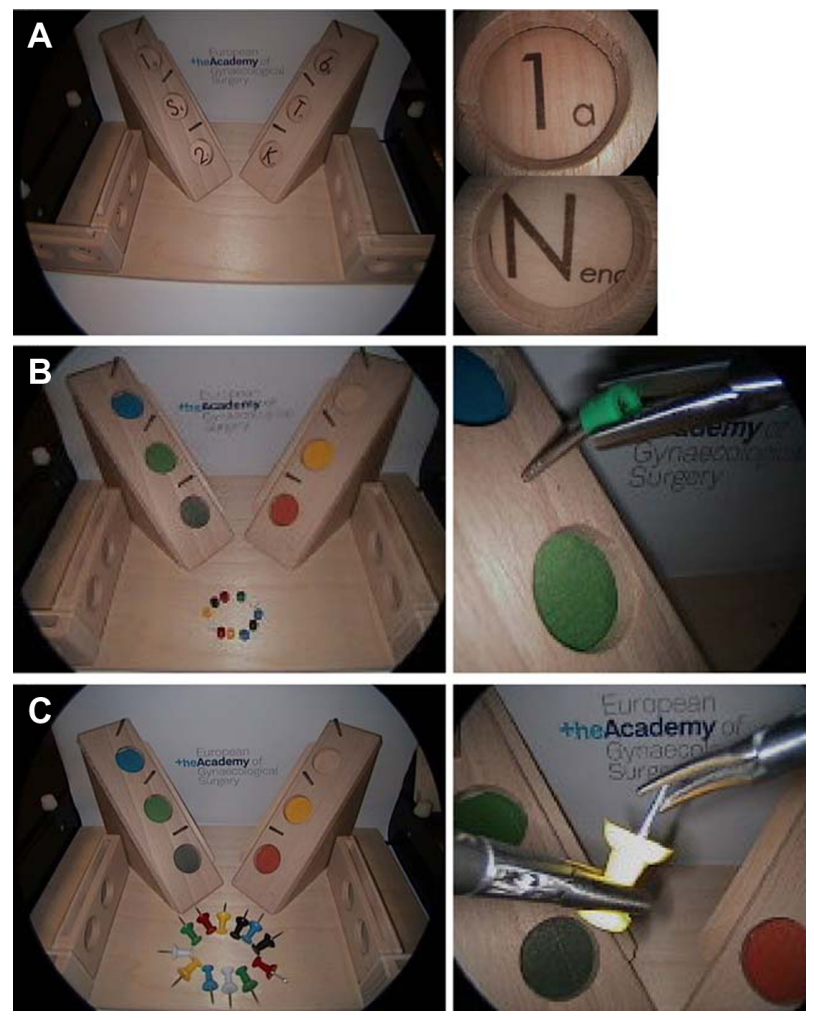

Figure 2 Laparoscopic psychomotor skills training and testing model. (A) EI, camera navigation. (B) E2, hand-eye coordination. (C) E3 bimanual coordination. Note: Springer and Gynecological Surgery. Campo R. A valid model for testing and training laparoscopic psychomotor skills. Gynecol Surg. 2010;7(2): I33-141. With kind permission from Springer Science and Business Media. ${ }^{45}$
On day 1 of instruction, students were introduced to the laparoscopy tools. The students were then trained in LASTT exercise E1 (camera navigation), in which they learnt to navigate a laparoscopic camera with a $30^{\circ}$ lens. On day 2 , students performed LASTT exercise E2, in which they practiced using the camera with the non-dominant hand and the forceps with the dominant hand and vice versa, in order to develop and test hand-eye coordination.

From day 3 onwards, the students worked in pairs, with one performing the exercise and the other holding the camera. Students developed communication and cooperation skills, especially in LASTT exercise E3, which taught bimanual coordination. The students also learned to handle laparoscopic forceps simultaneously with the dominant hand and the non-dominant hand.

On day 4, the students learned to handle a needle with a needle holder, still working in pairs. This involved doing a paperclip exercise, which consists of capturing a paperclip with the non-dominant hand grasper and, with a pronationsupination movement of the needle holder (dominant hand), penetrating it with a fixed needle and placing the paperclip in a box with a supination-pronation movement of the needle holder. During the needle rotation exercise, the student learns to grasp the needle tip with the non-dominant hand grasper and to turn the needle with the suture and the needle holder (dominant hand) over $360^{\circ}$ to put the needle in the needle holder simulating the different angles of surgical approaches.

\section{Block 2: intracorporeal suturing and knot tying}

This training block follows a multiple-stage approach to learning laparoscopic suturing. Most exercises are performed in the Szabo trainer box as described above.

On day 1, students learned to place the needle correctly in the needle holder, hold the needle in the dominant hand, and penetrate a skin pad (Limbs and Things, Bristol, UK) correctly. A specific trajectory needed to be followed. After this exercise, students learned to make a double throw around the dominant and non-dominant hand instruments. Polyfilament sutures were used.

At the beginning of the session on day 2, the squareto-slip principle, as described by Szabo et al, was learned by the students. ${ }^{16}$ The students then combined the learnt exercises to stitch and tie a complete knot using 3-0 Vicryl polyfilament sutures. After completing a knot, the result was inspected by the instructor. Cumulative time to complete a correct slipping knot was recorded by the students to selfmonitor their progress.

On day 3, the last exercise of the previous session was repeated, but this time with monofilament sutures. 
Polyfilament sutures are easier to handle during throwing because they have less memory, whereas monofilament sutures make it easier to create a slipping knot. All previous exercises were done with a fixed camera. Each student had his or her own working equipment. From the next exercise on, students worked in pairs, with one holding the camera while the other performed the suture. A $5 \mathrm{~cm}$ chicken skin incision had to be closed with a 4-0 Prolene polyfilament suture.

The day 4 session focused on suturing and knot tying under traction. For these exercises, a porcine stomach is installed in the pulsatile organ perfusion trainer without connecting to the pulsatile pump. The esophagus is connected to a fixed plug in the training box. A $3 \times 2 \mathrm{~cm}$ hole is created in the stomach fundus, and the student has to close this hole with a slipping knot while a colleague holds the camera. After completing this exercise, students were asked to create a fundoplication by wrapping the fundus of the stomach around the esophagus and suturing it.

\section{Block 3: tissue manipulation, dissection, and hemostasis}

During this block, all students worked in pairs. The operator performed the exercise while the assistant navigated the $30^{\circ}$ camera and could assist with one extra instrument depending on the instructions of the operator. Communication between the operator and assistant was encouraged. All exercises were performed in the pulsatile organ perfusion trainer, which is essentially a plastic box covered with neoprene. ${ }^{17}$ Basically, this is a plastic box covered with neoprene. By filling the box with $\mathrm{CO}_{2}$, a pneumoperitoneum can be simulated and students can insert trocars into the desired spaces. Animal tissue can be placed in the box, and when the main artery of the animal tissue is cannulated, the specimen can be connected with a pulsatile pump, pumping a red-colored fluid into it. When a blood vessel is damaged, bleeding is simulated (Figure 3). Monopolar and bipolar instruments are available. The goal of this training block was not to learn the specific steps of these procedures, but to give the students basic knowledge and feeling of tissue manipulation, hemostasis, and dissection. The following exercises were performed:

- An appendectomy model on day 1 , in which a rabbit bowel specimen is placed into the pulsatile organ perfusion trainer without connection to the pulsatile pump. Each student attempted to find the appendix, identify its mesentery, and perform an appendectomy using ligation loops.

- A gallbladder dissection on day 2, in which a porcine liver with gallbladder is placed into the pulsatile organ

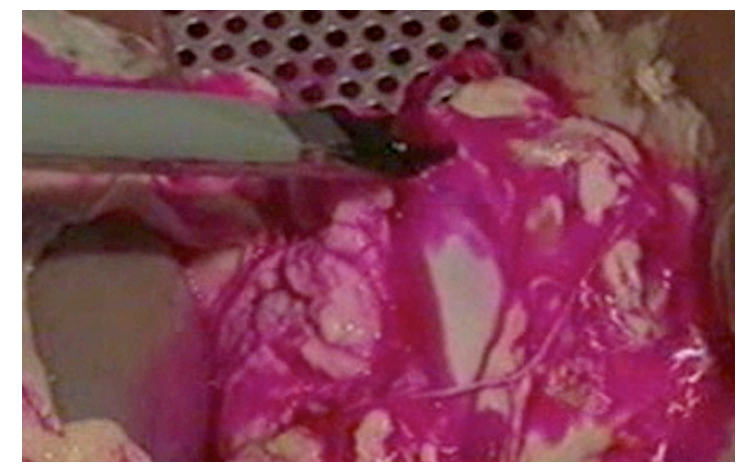

Figure 3 Pulsatile organ perfusion model of bleeding.

perfusion trainer. Students tried to dissect the gallbladder from the liver bed without damaging the liver bed or perforating the gallbladder. At this stage, the organ specimen is not connected to the pulsatile pump and the triangle of Callot is not dissected.

- A nephrectomy on day 3, in which a porcine kidney aorta specimen is placed into the pulsatile organ perfusion trainer and connected to the pulsatile pump. A perfusion line is inserted into the proximal aorta and the aorta is closed distally. Students had to dissect the renal artery and vein from the renal hilus to the aorta and vena cava without causing bleeding. After dissecting, students had to clip and cut the renal vessels.

- On day 4, students learned to perform an endoscopic resection, where chicken hearts are sutured in different places the inside of a pig's bladder to simulate papillary tumors. A resectoscope is entered in the pig's bladder and fixed with a zip-tie. Students learn to remove the chicken hearts using the resectoscope and electrocautery.

\section{Preparation of animal material}

The chicken material used were bought from a regular food shop the day before the training session and kept cool in a refrigerator. Porcine stomachs, kidneys, bladders, and livers were prepared and cleaned at a regular abattoir, vacuumpacked, and deep-frozen. The cannula preparation for the kidney model was done before freezing the specimen. Rabbit bowel specimens were taken from experimental rabbits at the end of the rabbit experiment, cleaned, and frozen. Specimens were taken out of the freezer the evening before the training session and allowed to thaw.

\section{Subjects}

All final year medical students applying for a surgical discipline (general surgery, urology, orthopedic surgery) were eligible for the course. 


\section{Assessment}

Before the start of the training sessions, a basic assessment of all students was performed. Three Southwestern drills (checkerboard, bean drop, and rope passage) were tested three times. ${ }^{18}$ The time taken to succeed in these tests was recorded. The mean of these three attempts was considered as the basic score for each student. Two weeks after the last training session of each block, these basic psychomotor exercises were evaluated again. The specific evaluations for each training block are as follows.

\section{Block I}

The LASTT E3 exercise was tested five times, with the camera navigated by the instructor. The minimum level to pass block 1 was a stable score around the plateau level (mean 98 [range 85-117] seconds) of the student's learning curve, as previously described. ${ }^{14}$

\section{Block 2}

A five $\mathrm{cm}$ chicken skin incision with a tissue gap of $2 \mathrm{~cm}$ between the edges had to be closed with three knots using the learned suturing technique and three separate polypropylene 4-0 sutures of $18 \mathrm{~cm}$. A fixed camera was used. Cumulative time to connect the skin edges adequately was used as the endpoint. When the skin edges were not approximated adequately or a loose knot was produced, redoing the suture was necessary. This allowed measurement of one parameter only (time) and assumed that errors and economy of movement would be reflected in this parameter. For this reason, time was an endpoint parameter for qualitative and quantitative analysis. The complete exercise (three knots and sutures) had to be done correctly in less than 13 minutes and 50 seconds. This was based on our previous research and corresponded with the plateau level of the learning curve measured in students who followed an intensive 6-day suturing course, each day comprising 1.5 hours of training in laparoscopic suturing. ${ }^{19}$

\section{Block 3}

Students performed the nephrectomy exercise, and their performance was recorded for video analysis by a single blinded observer. Scoring was done using a combined OSATS (Objective Structured Assessment of Technical Skills) and GOALS (Global Operative Assessment of Laparoscopic Skills) rating system. These validated evaluation scales have been shown to distinguish a beginner from a trained operator. ${ }^{20}$ For the botch scale, each item on the scale is ranked from 1 to 5 . A minimum of 30 points is required to achieve a positive overall evaluation.

\section{Evaluation}

The Southwestern drills were repeated before evaluation of each training block. All students rated the course on a 10-point VAS. They were asked if the course was useful, if they felt their skills progressed as a result of training, if their self-confidence had improved, if they thought a course like this should be obligatory, and if they would recommend this course to peers.

The performance of the students who completed our CST PTP course at the beginning of their registrar training was compared with that of registrars from the previous year who did not receive this training. The laparoscopic psychomotor skills of our students before the start of their CST PTP training were compared with the scores of residents before they started their first year. Their suturing skills were also compared. This was done by performing a procedure on a pig aorta collected from an abattoir. During this procedure, a $5 \mathrm{~mm} \times 40 \mathrm{~mm}$ incision was made. This hole had to be closed with three $18 \mathrm{~cm}$ polypropylene $4-0$ sutures. Prior to evaluation, an instructional video and text were studied for 30 minutes. Time to complete the procedure and number of movements were scored for each knot. The average result for the three knots was used in the statistical evaluation.

\section{Results}

Twenty-two final year medical students aged 24-26 years and applying for general surgery $(n=13)$, urology $(n=4)$, and orthopedic $(n=5)$ specialties participated in the course. There were six female students and 16 male students. Three students were left-handed and the others were right-handed. All students except one participated in every training session. This one student did not follow three of the lessons because of personal problems, but did participate in deliberate practice and did perform the skill tests.

The time score on the three Southwestern drills decreased significantly from the initial pretesting score to the evaluation score for block 1 (Figure 4). Only four students (18\%) passed block 1 during the first evaluation. Mean time for laparoscopic forceps handling and bimanual coordination (LASTT model) during this first evaluation was 196 (range 97.4-279.2) seconds. All 18 students passed the second evaluation, taking a mean 101.6 (range 94-115) seconds.

The mean time for a chicken skin suture during the first evaluation of block 2 was 281 (range 127-682) seconds. Six students (27\%) did not succeed during the first suturing evaluation and needed more intensive deliberate practice in laparoscopic suturing. Two students needed a third evaluation before being allowed to start training block 3 . One student 

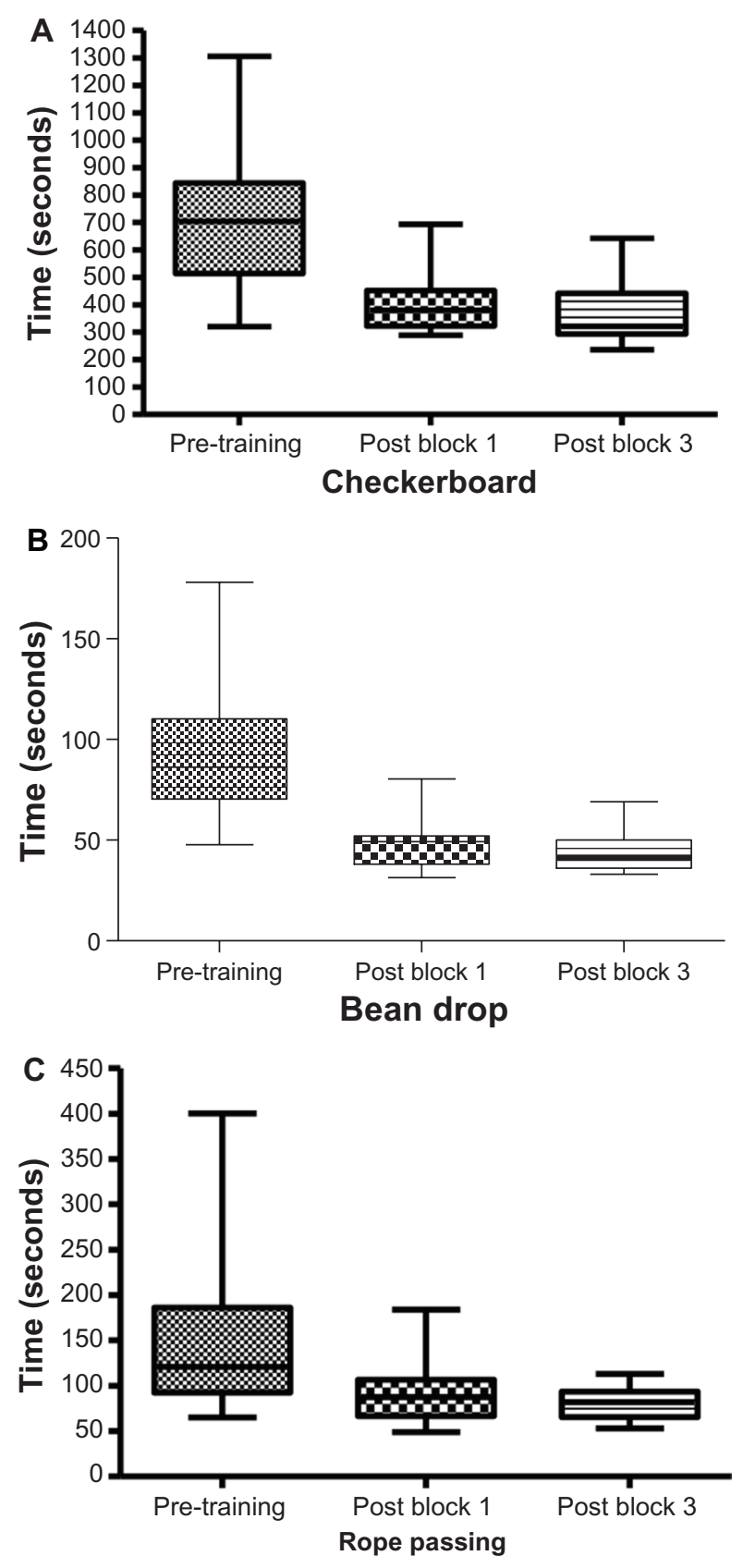

Figure 4 Improvement of Southwestern drills between different training sessions. Notes: Results on Southwestern drills before training, during evaluation of block 1 (psychomotor training), and during the final evaluation, after block 2 (suturing) and block 3 (dissection, tissue feeling, and hemostasis). There is a significant decrease in time needed to complete all three drill exercises after the first training block $(P<0.000 \mathrm{I})$. The second and third training block do not decrease the time needed further $(P>0.05)$.

did not succeed in block 2 and was not allowed to start the next training block. The mean score for evaluation of block 3 was 32 points.

All students performed deliberate practice. Mean deliberate practice time before evaluation varied from 40 minutes for block 1, to 2 hours and 10 minutes for block 2, to 2 hours and 23 minutes for block 3 . The students who did not succeed in the first evaluation test of block 1 spent, on average, an extra 45 minutes in the laboratory practicing, while the students who did not succeed in block 2 spent an extra 1.5 hours in the laboratory practicing. The average time spent on training (time spent during the course and deliberate practice time included) was 23 hours.

All students rated this course as being highly useful (VAS score 10). Students also believed that their skills progressed (mean VAS score 9), and that their self-confidence was better after the course (mean VAS score 7). All students were prepared to recommend this course to their peers and most of them thought that a course such as this should be compulsory (Figure 5).

There was no difference in psychomotor skills between our students before their training and the first year residents from the previous year. However, when our students were assessed at the beginning of their first year as registrars five months later, the preclinically trained students outperformed the registrars who did not receive the preclinical training on the Southwestern drills $(P<0.0001)$.

Further, on the suturing evaluation using a pig aorta, the baseline level for the preclinically trained students was clearly better than for the ones who did not receive previous training. Although a more difficult task was tested (suturing porcine aorta instead of chicken skin) for the CST PTPtrained students, there was an important skill decay for this more difficult task (Figure 6).

\section{Discussion}

It has been suggested that simulation-based training allows for development of the "pre-trained novice", ie, an individual trained to the point where many psychomotor skills

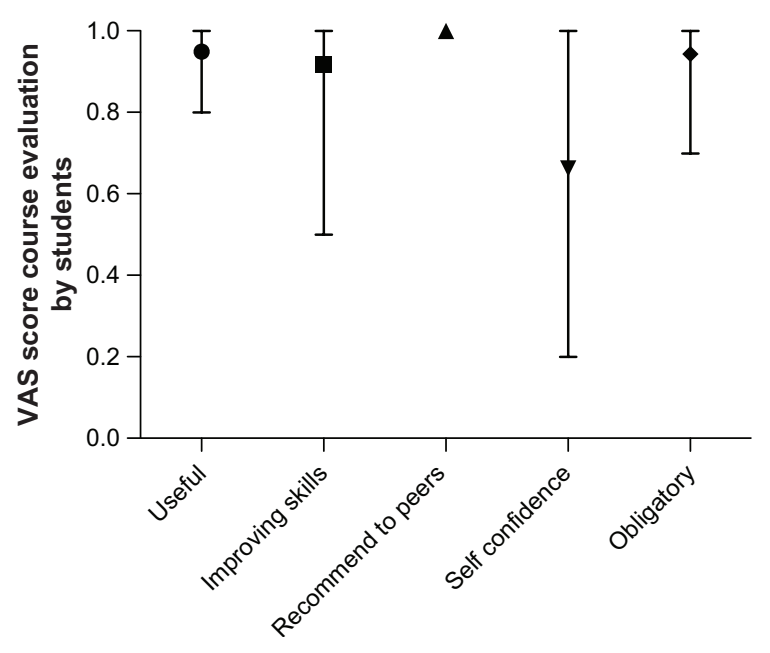

Figure $\mathbf{5}$ Course evaluation by students' visual analog score. 

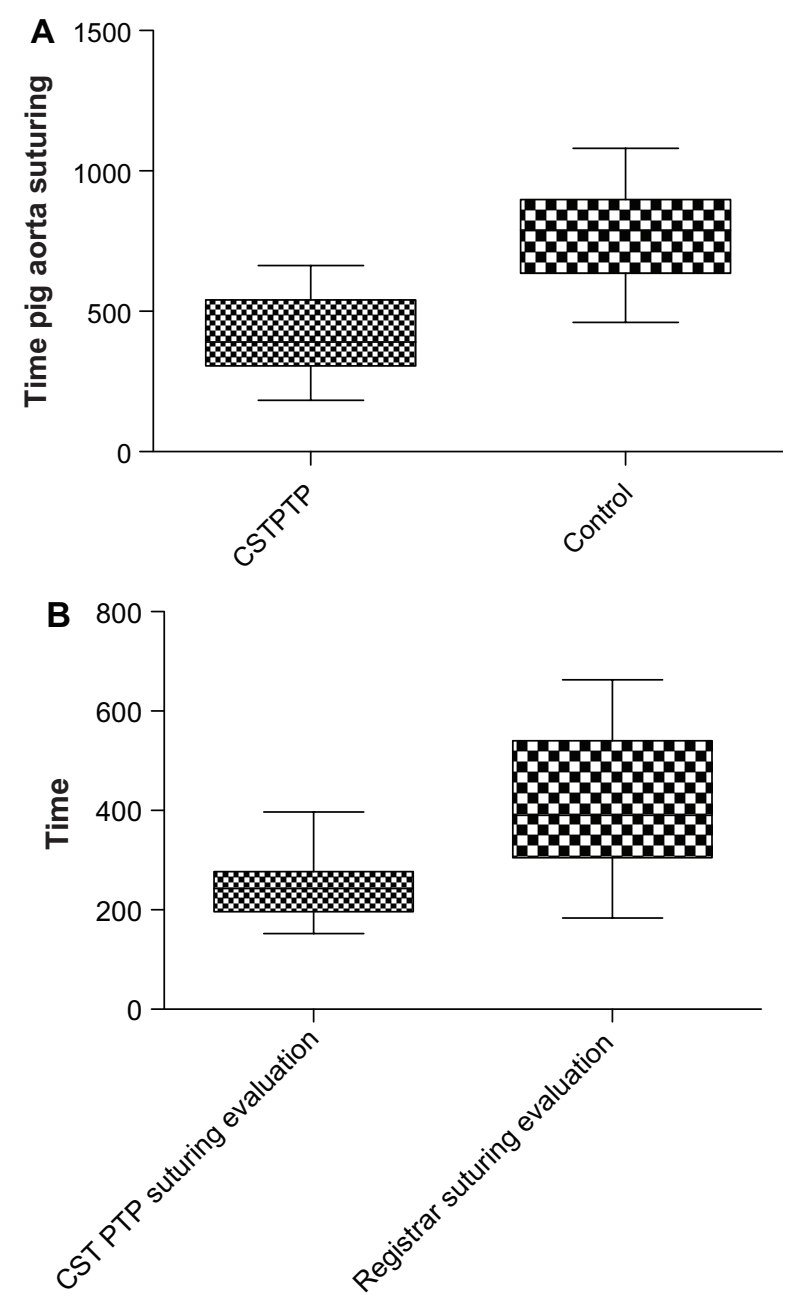

Figure 6 (A) At the beginning of surgical residency, the preclinical trained students outperformed the registrars from the previous year on suturing a pig aorta, a task that none of the trainees had performed before $(P<0.000$ I, $t$-test). (B) However, when the suturing evaluation for students who completed the preclinical training program at the Centre for Surgical Technologies after block 2 (suturing chicken skin) was compared with the more complex suturing evaluation at the start of their residency (suturing pig aorta), a marked skill decay is seen $(P=0.0055$, paired t-test).

and spatial judgments have been automated, enabling the surgeon-in-training to focus more on learning operative strategy and how to handle intraoperative complications, than wasting valuable operating room time on initial refinement of psychomotor skills. With adequate pre-training, the trainee can gain maximum advantage from the supervised opportunities for training in the operating room or endoscopy suite. ${ }^{21}$

It was our goal to develop a preclinical laparoscopy course for medical students appropriate to their intended surgical specialty in order to equip them with the laparoscopy skills necessary to start their residency. Rather than focusing on one model, we strove to develop a holistic training program integrating several different simulators. To do so, existing evidence on educational models using simulators was referenced, and we also incorporated input from trainers and trainees on particular issues they wanted addressed.

Limited feedback from experts is also an important factor in training progression. However, it is important to note that intense expert feedback is known to inhibit certain intrinsic learning strategies and problem-solving activities in the student, resulting in dependency on feedback provided and inferior performance when that feedback is withdrawn after training. ${ }^{22,23}$

However, internal feedback, ie, the feedback a trainee receives himself or herself as a natural consequence of performance, is an important factor in skill retention. Therefore, it is important that trainees be able to evaluate their own results in a goal-directed training program. ${ }^{22,24,25}$ Simple evaluation systems for each exercise were used to allow for this process of internal development.

Our results show that it is feasible to develop and implement a preclinical laparoscopy course for medical students, incorporating different models and scientific evidence within training. All students were highly satisfied and believed that their skills improved and prepared them for their residency. Even the student who did not succeed in the block 2 evaluation and could not start the block 3 training thought this course was important to prepare students for a surgical residency. Although our performance goals were not set as selection criteria, the aforementioned student who did not pass chose to apply for another specialty.

It was also shown objectively that laparoscopic skills in these novices improved during the course and that they retained their learned skills, at least until the start of their surgery residency program. Six months after the course, at the beginning of their residency, our pre-trained students transferred their skills to a new and more difficult suturing task and performed even better at the beginning of their residency than a similar group of residents with similar psychomotor skills who had not been trained before. Therefore, our pretrained medical students could benefit by having a head start in their residency programs.

Block 1 psychomotor training improved the score on the Southwestern drills. These drills have been shown to improve basic clinical laparoscopy skills. ${ }^{18}$ The Southwestern results reached after block 1 psychomotor training were comparable with the scores reached in our previous study in which students learned intracorporeal suturing. ${ }^{19}$ Southwestern scores did not improve any further after blocks 2 and 3, indicating that the lowest level was reached or that when a trainee has a certain psychomotor level, further training with suturing practice or dissection practice cannot be scored using these 
basic drill exercises. In the following section, we discuss the different elements of our training program.

\section{Content of training and different types of simulators}

When embarking on laparoscopy training, the trainee must first overcome some psychomotor difficulties. Therefore, the first element of the training consists of psychomotor exercises. This type of exercise has been shown to be transferable to the clinic. ${ }^{18}$ Furthermore, Southwestern drills have been shown to decrease training time and cost for the American proficiency-based fundamentals of laparoscopic surgery training. ${ }^{26}$ Also, the hand-eye coordination exercises in our LASTT model have been shown to facilitate acquisition of laparoscopic intracorporeal suturing skills. ${ }^{27}$

As a feasible and validated tool, we preferred the LASTT method over the American Fundamentals of Laparoscopic Surgery training program because the validation process of this model was done in our laboratory, ${ }^{14,15}$ so much of the validation data and learning curves were available and therefore, models were easily accessible.

Both the dominant hand and non-dominant hand need to be trained. Previous research had indicated that dominant hand skills are better than non-dominant hand skills, and that changes in the non-dominant hand are greater than changes in the dominant hand during training. Therefore, fully training the non-dominant hand is advisable for acquiring an optimal level of skill. ${ }^{27,28}$ Laparoscopic suturing and knot tying is considered by many surgeons to be an advanced skill. However, Aggarwal et al has shown that endoscopic suturing can be learned in the laboratory by trainees during skill courses, regardless of their baseline laparoscopic experience. ${ }^{29}$ Teaching laparoscopic suturing to juniors seemed to be feasible and efficacious. Furthermore, suturing is sometimes required unexpectedly, even during the most simple and straightforward procedures.

Tissue feeling and manipulation as well as basic electrocautery techniques during laparoscopic surgery differ from those in open surgery. Originally, these skills were learned in animal models and practiced on rabbits or pigs. However, the cost and ethics involved in practicing surgical techniques on animals under anesthesia are troublesome. While practice on animal models may be useful for learning specific advanced surgical techniques, it was our aim to teach general tissue feeling, dissection, and electrocautery techniques, all skills that are useful in any type of surgery.

We did not use virtual reality trainers. Current evidence does not show a clear advantage of either virtual reality or synthetic models in teaching technical skills, especially not for training psychomotor skills. Most virtual trainers teach a specific procedure, incorporate feedback, and can give structured training and explanation. It was not our aim to teach a specific procedure but rather to teach general skills that are useful for different types of surgical procedures. Therefore, we needed to use different dissection models. If we wanted to incorporate virtual reality, we would have had to use different virtual reality trainers, which would have further increased the costs. Evidence comparing virtual reality training with animal training is lacking. The pulsatile organ perfusion trainer seemed to us to be the best available alternative.

During our previous training sessions organized for junior doctors at our center, we used pig models, rabbit models, and pulsatile organ perfusion trainers. Our students were always enthusiastic about working on the pulsatile organ perfusion trainer, mainly because they were able to make mistakes using these trainers and could repeat the same dissection exercise without the need for a new animal.

Many students, especially those interested in urology and orthopedics, were also interested in acquiring endoscopic skills. Therefore, instruction was not only in laparoscopic skills, but in basic endoscopic skills as well. A correlation between endoscopy and laparoscopy learning had been postulated elsewhere. ${ }^{30}$

\section{Training structure}

A recent review of skill transferability stated that it is not only the simulator but also the mode of training on the simulator that determines the degree of transference of skill to the operative setting. Therefore, during the development of our course, we implemented recent findings about training structure. ${ }^{3}$

\section{Modeling}

Pearson et al showed that a structured explanation of the skill to be learnt is important. ${ }^{31}$ More recently, Alk et al indicated that use of a teaching video illustrating the principles of laparoscopic suturing is an effective method for acquisition of basic laparoscopic suturing skills. ${ }^{32}$ Video tutorials before and during training have been shown to lead to superior training. ${ }^{33}$ Stefanidis et al showed that limited instructor feedback is more effective than intense feedback during training, coupled with video tutorials, limited feedback accelerated learning, and improved resource utilization by minimizing the need for instructor involvement. ${ }^{22}$ Also, in an earlier study, Rosser et al demonstrated that a CD-ROM tutorial on laparoscopic skills effectively transferred the cognitive information necessary for skill development. ${ }^{34}$ 
To give students access to the instructional videos, which were specifically designed for this course, we integrated these videos into a personalized web-based module developed specifically for e-learning at the University of Leuven. ${ }^{13}$ This made it possible for students to prepare for the upcoming training session and to review the different steps of the skills to be learned at their convenience.

\section{Training frequency}

We and others have shown that short training sessions distributed over time result in better training performance and retention than massed practice. ${ }^{19,35}$ However, training courses in most education centers are still organized as intensive one-day or two-day courses. Residents are involved in daily clinical work in different hospitals, making it difficult to organize and attend short daily courses. Furthermore, distributed training under expert supervision requires a considerable faculty time commitment and is subject to scheduling conflicts. In a recent randomized controlled study of medical students learning intracorporeal suturing, it was shown that video demonstrations combined with peer feedback can partly replace expert supervision. ${ }^{23}$ Skill coaches also appear to be valid assessors of trainee performance. ${ }^{36}$ In addition, final medical students follow daily lessons at the university. Switching a laparoscopy course from residency to the last year of medical school makes it easier to organize the course in an evenly distributed way.

\section{Deliberate practice}

The concept of deliberate practice for acquiring expert performance is well validated in many domains, including sports and music. ${ }^{37}$ Similarly, the concept of deliberate practice translates into better performance in surgical education. ${ }^{38}$ Deliberate practice allows students to focus their training on defined tasks in a purposeful manner. This technique involves repeated practice with immediate feedback on performance. It appears that the construct of deliberate practice inherent in proficiency-based training provides an additional extrinsic motivational factor that engages learners and leads to enhanced learning outcomes. ${ }^{39}$

Previous research has shown the importance of deliberate practice on skill transfer and skill retention. ${ }^{38}$ However, on the other hand, a study by Chang et al found that, when given the opportunity to train in a simulation laboratory, many residents chose not to use it for reasons relating to time, location, and/or lack of interest. To increase participation rates and make simulation an effective part of the training curriculum, the authors suggest that simulation-based training be made mandatory and integrated fully into the curriculum. ${ }^{40}$

Van Dongen et al also found that free unlimited access to a virtual reality simulator without any form of obligation or assessment did not motivate surgical registrars to use the simulator. Introducing a competitive element had only a marginal effect. They concluded that this access to training models for deliberate practice is only effective when it is an integrated and mandatory part of the surgical curriculum. ${ }^{41}$

In our previous study of laparoscopic suturing in which deliberate practice was not mandatory, only half of the students practiced in this manner. ${ }^{19}$ However, in the present study, we found that deliberate practice was done intensively by all our trainees with an approximate mean time of two hours per training block. Students were given performance criteria to reach before being allowed to start the next training block. Performance goals have been reported as being important for motivating students engaged in deliberate practice. ${ }^{3}$

The mean time for deliberate practice was low only after block 1, possibly because of the more abstract nature of the tasks learnt. Consequently, only four students succeeded in the first evaluation of block 1. After intensifying the time spent in the training laboratory, all of the students passed the evaluation. During psychomotor training, deliberate practice for psychomotor exercises will need more external motivation, which might be done by organizing psychomotor skill competitions. Residents are forced to balance their clinical duties with instructional time. While responsibilities often impinge on the opportunity for deliberate practice, medical students are usually highly motivated to participate in skills development courses.

\section{Proficiency-based training}

Proficiency-based training allows for variation between students in the amount of training time and number of practice trials needed, while keeping mastery constant. Because the goal of proficiency training is to bring students to the same ultimate skill level, proficiency-focused training accommodates the ability and development of each individual. Therefore, the training regimen is based more on the capabilities of the individual than outside influences, such as time or number of practice trials. Stefanidis et al suggest that proficiency-based training leads to better laparoscopic skill acquisition and that skills learnt in such a way transfer to the operating theater and lead to better skill retention. ${ }^{42}$ However, how proficiency is defined is ambiguous. The initial performance level of experts on a certain task is often set as a cutoff level that "proficiency" level trainees have to reach. ${ }^{8,43}$ In our program, time 
scores were used for basic psychomotor skills and suturing. Time proved to be an easy assessment parameter that made it easy for students to continue along their own learning curve. One hundred percent efficacy was demanded for each repetition (ie, the repetition was considered to be complete only after successful performance of the exercise), assuming with some limitations that any mistake would be reflected in the main parameter, ie, time. When mistakes were made, time went on, making time an effective predictor of skill performance. This system is objective, tutor-independent, and useful for self-assessment.

While many studies have taken the first expert score as the proficiency criterion, we took the plateau level of the student's learning curve as the proficiency criterion if this level was better than the first expert score. Our previous research (albeit not as yet published) indicated that, for many psychomotor exercises, after a few repetitions, new students could improve beyond the first expert score, but after further training, the student learning curve and expert learning curve came to a plateau which was different. The student plateau was often better than the initial expert score, but the expert plateau was reached more rapidly than the student plateau. To help ensure that achievement of this proficiency level was not a random event during evaluation, three consecutive repetitions at or better than the required level were needed to demonstrate that the skill had been acquired.

Many students did not pass the first evaluation of block 1 . One of the reasons for this, as mentioned above, was the comparatively short time spent in performing deliberate practice. However, our performance score was set at the plateau level of the student learning curve, which might indicate more of a mastery level then a proficiency level. Mastery training is in line with the concept of overtraining, and in the motion learning literature, it is believed to be an important parameter for skill transfer and skill retention, ${ }^{33}$ and a recent study of laparoscopic psychomotor training has not demonstrated that overtraining psychomotor exercises improves skill retention. ${ }^{8}$ However, ours is only one study and it might be that our performance goals for the first training block were too high.

Only one kidney dissection had to be done for evaluation of block 3. The reason for this decision was that a global rating scale was used instead of time scores. This global rating score provides a more continuous evaluation. A median score of three on each section of the global rating scale had to be reached. We agree that OSTAS and GOALS do not allow students to perform self-assessment. However, deliberate practice for dissection exercises must always be done in pairs. Therefore, peer feedback was always available.

We also agree that virtual reality systems incorporating feedback could have been used to set performance scores, but these expensive systems were not used. Although we tried to keep the group homogenous, students without classic basic surgical training probably have a wide range of surgical endoscopic skills. The long-term efficacy of this course and its transferability to the clinic is still not proven. However, our expectations are quite positive. The drawbacks to our suggested mode of instruction are financial resources and the demands on faculty and student time. It was only thanks to financial funding from KU Leuven, and their educational research, development, and implementation projects, that we could organize this training. All models used are inexpensive and flexible in use. Although our suggested training program requires a significant amount of faculty time in the laboratory, peer feedback could partly replace faculty feedback. ${ }^{23}$

An important factor in developing a training program like this is protected educational time. For this course, an average of 23 hours was necessary to complete the training. This is comparable with three full-day courses organized in typical training centers; however, because of the specific setup with distributed sessions and deliberate practice, it might be more difficult to organize this sort of scheduled training for surgical registrars. Therefore, it may be easier to teach this course to final year medical students.

At the moment, it is not clear when interval training sessions to keep residents skills up to date need to be organized. A recent study by Maagaard et al showed that novices retained their skills at six months, but by 18 months the skills had returned to the pre-training level. ${ }^{44}$ That study also indicated that novices' skills deteriorated between six and 18 months, whereas experts showed consistency over time. Our students start their residency training sooner than six months after the end of training, which should minimize the loss of already acquired skills. If or when skills deteriorate when students are exposed to assisting laparoscopic surgery must still be defined.

Future courses will also include gynecology residents because they are increasingly required to perform laparoscopic procedures. We believe that a course like ours will prepare medical students for their residency more effectively than existing courses. The substantial laparoscopic and endoscopic skills medical students will have developed before their residency will make it possible for them to focus more on operative technique then on basic skills when they assist in surgical procedures. However, it still needs to be demonstrated that 
our course can influence the clinical learning curve. This will be a topic of future research at our center.

\section{Acknowledgments}

Many thanks to Ann Lissens and Ivan Laermans from the Centre of Surgical Technologies who were a great help organizing these training sessions and evaluations, and to all the students who volunteered for this project, which was funded by a grant from KU Leuven, University of Leuven, Educational Research, Development and Implementation Projects (OOI 2005/39). Authors would also like to thank Robi Struyf for his assistance with Figure 1.

\section{Disclosure}

GDW, SVB, and CA have no conflicts of interest or financial ties to disclose. DDR is on the speaker bureaus of Astellas, Pfizer, AMS, Medtronic, Allergan, and Bard, and is an investigator for Astellas, Pfizer, AMS, Xention, Allergan, Gynecare, Dynamesh, and Bard. He has done consultancy work for Astellas, Pfizer, AMS, Xention, Medtronic, Allergan, and Bard, but did not receive research grants related to this publication from Astellas, AMS, Medtronic, or Bard.

\section{References}

1. Cauraugh JH, Martin M, Martin KK. Modeling surgical expertise for motor skill acquisition. Am J Surg. 1999;177(4):331-336.

2. Subramonian K, DeSylva S, Bishai P, Thompson P, Muir G. Acquiring surgical skills: a comparative study of open versus laparoscopic surgery. Eur Urol. 2004;45(3):346-351.

3. Sturm LP, Windsor JA, Cosman PH, Cregan P, Hewett PJ, Maddern GJ. A systematic review of skills transfer after surgical simulation training. Ann Surg. 2008;248(2):166-179.

4. Palter VN, Grantcharov TP. Simulation in surgical education. CMAJ. 2010;182(11):1191-1196.

5. Al-Kadi AS, Donnon T, Oddone Paolucci E, Mitchell P, Debru E, Church N. The effect of simulation in improving students' performance in laparoscopic surgery: a meta-analysis. Surg Endosc. 2012;26(11):3215-3224.

6. Cannon-Bowers JA, Bowers C, Procci K. Optimizing learning in surgical simulations: guidelines from the science of learning and human performance. Surg Clin North Am. 2010;90(3):583-603.

7. Stefanidis D, Arora S, Parrack DM, et al. Research priorities in surgical simulation for the 21st century. Am J Surg. 2012;203(1):49-53.

8. Kolozsvari NO, Kaneva P, Brace C, et al. Mastery versus the standard proficiency target for basic laparoscopic skill training: effect on skill transfer and retention. Surg Endosc. 2011;25(7):2063-2070.

9. Anastakis DJ, Wanzel KR, Brown MH, et al. Evaluating the effectiveness of a 2-year curriculum in a surgical skills center. Am J Surg. 2003;185(4):378-385.

10. Schout BM, Hendrikx AJ, Scheele F, Bemelmans BL, Scherpbier AJ. Validation and implementation of surgical simulators: a critical review of present, past, and future. Surg Endosc. 2010;24(3):536-546.

11. Ritter EM, Scott DJ. Design of a proficiency-based skills training curriculum for the fundamentals of laparoscopic surgery. Surg Innov. 2007;14(2):107-112.

12. Edelman DA, Mattos MA, Bouwman DL. Impact of fundamentals of laparoscopic surgery training during medical school on performance by first year surgical residents. J Surg Res. 2011;170(1):6-9.
13. Buelens H, Van Rentergem L, Verhesschen P, Gaeremynck J, Mennes K. Invoeringsstrategie van een digitale toets- en leeromgeving. Het Toledoproject aan de K.U.Leuven. [Implementing a digital testing and learning environment: The KU Leuven Toledo- project]. In: D’Haese I, Valcke M, editors. Digitaal leren. ICT-toepassingen in het hoger onderwijs [Digital Learning. ICT applications in higher education], 1st ed. Leuven, Belgium: LannooCampus; 2005. Pages 243-264. Dutch.

14. Molinas CR, Win G, Ritter O, Keckstein J, Miserez M, Campo R. Feasibility and construct validity of a novel laparoscopic skills testing and training model. Gynecol Surg. 2008;5(4):281-290.

15. Campo R, Reising C, Belle Y, Nassif J, O’Donovan P, Molinas CR. A valid model for testing and training laparoscopic psychomotor skills. Gynecol Surg. 2010;7(2):133-141.

16. Szabo Z, Hunter J, Berci G, Sackier J, Cuschieri A. Analysis of surgical movements during suturing in laparoscopy. Endosc Surg Allied Technol. 1994;2(1):55-61.

17. Szinicz G, Beller S, Bodner W, Zerz A, Glaser K. Simulated operations by pulsatile organ-perfusion in minimally invasive surgery. Surg Laparosc Endosc. 1993;3(4):315-317.

18. Scott DJ, Bergen PC, Rege R V, et al. Laparoscopic training on bench models: better and more cost effective than operating room experience? J Am Coll Surg. 2000;191(3):272-283.

19. De Win G, De Ridder D, Van Bruwaene S, Miserez M. The optimal frequency of endoscopic skill labs for training and skill retention on suturing; a randomized controlled trial. J Surg Educ. 2013;70:384-393.

20. Vassiliou MC, Feldman LS, Andrew CG, et al. A global assessment tool for evaluation of intraoperative laparoscopic skills. Am J Surg. 2005;190(1):107-113.

21. Gallagher AG, Ritter EM, Champion H, et al. Virtual reality simulation for the operating room. Ann Surg. 2005;241(2):364-372.

22. Stefanidis D, Korndorffer JR, Heniford BT, Scott DJ. Limited feedback and video tutorials optimize learning and resource utilization during laparoscopic simulator training. Surgery. 2007;142(2):202-206.

23. Van Bruwaene S, De Win G, Miserez M. How much do we need experts during laparoscopic suturing training? Surg Endosc. 2009;23(12):2755-2761.

24. Xeroulis GJ, Park J, Moulton C-A, Reznick RK, Leblanc V, Dubrowski A. Teaching suturing and knot-tying skills to medical students: a randomized controlled study comparing computer-based video instruction and (concurrent and summary) expert feedback. Surgery. 2007;141(4):442-449.

25. Porte MC, Xeroulis G, Reznick RK, Dubrowski A. Verbal feedback from an expert is more effective than self-accessed feedback about motion efficiency in learning new surgical skills. Am J Surg. 2007;193(1):105-110.

26. Fried GM, Feldman LS, Vassiliou MC, et al. Proving the value of simulation in laparoscopic surgery. Ann Surg. 2004;240(3): $518-525$.

27. Molinas CR, Campo R. Defining a structured training program for acquiring basic and advanced laparoscopic psychomotor skills in a simulator. Gynecol Surg. 2010;7(4):427-435.

28. Elneel FHF, Carter F, Tang B, Cuschieri A. Extent of innate dexterity and ambidexterity across handedness and gender: Implications for training in laparoscopic surgery. Surg Endosc. 2008;22(1):31-37.

29. Aggarwal R, Hance J, Undre S, et al. Training junior operative residents in laparoscopic suturing skills is feasible and efficacious. Surgery. 2006;139(6):729-734.

30. Kneebone RL, Nestel D, Moorthy K, et al. Learning the skills of flexible sigmoidoscopy - the wider perspective. Med Educ. 2003;37 Suppl 1: $50-58$.

31. Pearson AM, Gallagher AG, Rosser JC, Satava RM. Evaluation of structured and quantitative training methods for teaching intracorporeal knot tying. Surg Endosc. 2002;16(1):130-137.

32. Akl MN, Giles DL, Long JB, Magrina JF, Kho RM. The efficacy of viewing an educational video as a method for the acquisition of basic laparoscopic suturing skills. J Minim Invasive Gynecol. 2008;15(4):410-413. 
33. Magill RA. Motor Learning. Concepts and Applications. New York, NY: McGraw Hill; 2001.

34. Rosser JC, Herman B, Risucci DA, Murayama M, Rosser LE, Merrell RC. Effectiveness of a CD-ROM multimedia tutorial in transferring cognitive knowledge essential for laparoscopic skill training. Am J Surg. 2000;179(4):320-324.

35. Mackay S, Morgan P, Datta V, Chang A, Darzi A. Practice distribution in procedural skills training: a randomized controlled trial. Surg Endosc. 2002;16(6):957-961.

36. Rooney DM, Hungness ES, Darosa DA, Pugh CM. Can skills coaches be used to assess resident performance in the skills laboratory? Surgery. 2012;151(6):796-802.

37. Ericsson K, Krampe R, Teschromer C. The role of deliberate practice in the acquisition of expert performance. Psychol Rev. 1993;100(3): 363-400.

38. Crochet P, Aggarwal R, Dubb SS, et al. Deliberate practice on a virtual reality laparoscopic simulator enhances the quality of surgical technical skills. Ann Surg. 2011;253(6):1216-1222.

39. Willis RE, Richa J, Oppeltz R, et al. Comparing three pedagogical approaches to psychomotor skills acquisition. Am J Surg. 2012;203(1): $8-13$.
40. Chang L, Petros J, Hess DT, Rotondi C, Babineau TJ. Integrating simulation into a surgical residency program: is voluntary participation effective? Surg Endosc. 2007;21(3):418-421.

41. Van Dongen KW, van der Wal WA, Rinkes IH, Schijven MP, Broeders IA. Virtual reality training for endoscopic surgery: voluntary or obligatory? Surg Endosc. 2008;22(3):664-667.

42. Stefanidis D, Korndorffer JR, Sierra R, Touchard C, Dunne JB, Scott DJ. Skill retention following proficiency-based laparoscopic simulator training. Surgery. 2005;138(2):165-170.

43. Stefanidis D, Acker C, Heniford BT. Proficiency-based laparoscopic simulator training leads to improved operating room skill that is resistant to decay. Surg Innov. 2008;15(1):69-73.

44. Maagaard M, Sorensen JL, Oestergaard J, et al. Retention of laparoscopic procedural skills acquired on a virtual-reality surgical trainer. Surg Endosc. 2011;25(3):722-727.

45. Campo R. A valid model for testing and training laparoscopic psychomotor skills. Gynecol Surg. 2010;7(2):133-141.
Advances in Medical Education and Practice

\section{Publish your work in this journal}

Advances in Medical Education and Practice is an international, peerreviewed, open access journal that aims to present and publish research on Medical Education covering medical, dental, nursing and allied healthcare professional education. The journal covers undergraduate education, postgraduate training and continuing medical education

\section{Dovepress}

including emerging trends and innovative models linking education, research, and healthcare services. The manuscript management system is completely online and includes a very quick and fair peer-review system. Visit http://www.dovepress.com/testimonials.php to read real quotes from published authors.

Submit your manuscript here: http://www.dovepress.com/advances-in-medical-education-and-practice-journal 